\title{
Complete spacelike hypersurfaces with positive $r$-th mean curvature in a semi-Riemannian warped product
}

\author{
Yaning Wang and Ximin Liu
}

\begin{abstract}
In this paper, by supposing a natural comparison inequality on the positive $r$-th mean curvatures of the hypersurface, we obtain some new Bernstein-type theorems for complete spacelike hypersurfaces immersed in a semi-Riemannian warped product of constant sectional curvature. Generalizing the above results, under a restriction on the sectional curvature or the Ricci curvature tensor of the fiber of a warped product, we also prove some new rigidity theorems in semi-Riemannian warped products. Our main results extend some recent Bernstein-type theorems proved in $[12,13,14]$.
\end{abstract}

\section{Introduction}

One of the basic problems on spacelike hypersurfaces is the problem of uniqueness of spacelike hypersurfaces with constant mean curvature, more generally, that of spacelike hypersurfaces with geometric condition which is characterized by higher order mean curvature. The aim of this paper is to study such type problem of spacelike hypersurfaces immersed in a semi-Riemannian warped product. Before giving details of our main results, we shall first present a brief outline of some recent papers concerning uniqueness theorems related to ours.

Key Words: Complete spacelike hypersurface, semi-Riemannian warped product, $r$-th mean curvature, Bernstein-type theorem.

2010 Mathematics Subject Classification: Primary 53C42; Secondary 53B30, 53C50, $53 Z 05$.

Received: May, 2013

Accepted: September, 2013. 
By applying a key lemma proved in [11], F. Camargo, A. Caminha and H. F. De Lima [12] proved some Bernstein-type theorems concerning complete and connected spacelike hypersurfaces in steady state-type spacetimes and hyperbolic-type spaces. Generalizing the above results, A. G. Colares and H. F. de Lima [13] obtained some rigidity theorems in semi-Riemannian warped products.

Later, by replacing null convergence condition by $(\ln f)^{\prime \prime} \leq 0$, L. J. Alílas, D. Impera and M. Rigoli [5] obtained uniqueness theorems concerning compact spacelike hypersurfaces with constant higher order mean curvature immersed in a spatially closed generalized Robertson-Walker spacetime. They also investigated the uniqueness of complete spacelike hypersurfaces by using a generalization of the Omori-Yau maximal principal. We also refer the reader to $[2,4]$ for some relevant results concerning higher order mean curvature.

Recently, by supposing a natural comparison inequality between the $r$-th mean curvatures of the hypersurface immersed in a semi-Riemannian warped product, H. F. de Lima and J. R. de Lima [14] proved a uniqueness theorem with the null convergence condition, i.e., $k \geq \sup _{I}\left(f f^{\prime \prime}-f^{\prime 2}\right)$. In fact, there is a little mistake in the proof of Theorem 1.1 of [14], we give the details about that mistake (see Remark 5.1) in section 5. Note that the conclusion of Theorem 1.1 in [14] still holds if we correct an inequality in the assumption of this theorem.

In this paper, following [2] and [5] we consider the action of the second order linear differential operator $L_{r}$ (see section 2) on the integral of the warping function, which makes us to obtain some more accurate estimates. It is worth to point out that the Laplacian of integral of the warping function was studied by the present authors in $[20,21]$ to obtain some uniqueness results. Throughout this paper, we denote by $\mathcal{L}\left(\Sigma^{n}\right)$ the space of Lebesgue integrable functions on spacelike hypersurface $\Sigma^{n}$. Then, by applying a result proved by CaminhaSousa-Camargo [11] and supposing a natural comparison inequality between the $r$-th mean curvature of a hypersurface, we obtain new Bernstein-type theorem (whose proof can be seen in section 4) as follows.

Theorem 1.1. Let $\bar{M}^{n+1}=-I \times_{f} M^{n}$ be a Lorentzian warped product of constant sectional curvature. Let $\psi: \Sigma^{n} \rightarrow \bar{M}^{n+1}$ be a complete and connected spacelike hypersurface with bounded second fundamental form and bounded away from the infinity of $\bar{M}^{n+1}$. Suppose that $H_{r}$ and $H_{r+1}$ are positive for some $1 \leq r \leq n-1$ such that

$$
\frac{H_{r+1}}{H_{r}} \geq \frac{f^{\prime}}{f}(h)>0 .
$$

If h has local minimum on $\Sigma^{n}$ and $|\nabla h| \in \mathcal{L}\left(\Sigma^{n}\right)$, then $\Sigma^{n}$ is a slice of $\bar{M}^{n+1}$. 
With regard to spacelike hypersurfaces in Robertson-Walker spacetimes, we also obtain the following rigidity result which generalizes the above Theorem 1.1 .

Theorem 1.2. Let $\bar{M}^{n+1}=-I \times_{f} M^{n}$ be a Robertson-Walker spacetime whose Riemannian fiber $M^{n}$ has constant sectional curvature $k$ satisfying

$$
k \leq \inf _{I}\left(f f^{\prime \prime}-f^{\prime 2}\right) .
$$

Let $\psi: \Sigma^{n} \rightarrow \bar{M}^{n+1}$ be a complete and connected spacelike hypersurface with the bounded second fundamental form and bounded away from the infinity of $\bar{M}^{n+1}$. Suppose that $H_{r}$ and $H_{r+1}$ are positive for some $1 \leq r \leq n-1$ and satisfy (1). If $h$ has local minimum on $\Sigma^{n}$ and $|\nabla h| \in \mathcal{L}\left(\Sigma^{n}\right)$, then $\Sigma^{n}$ is a slice of $\bar{M}^{n+1}$.

This paper is organized as follows. We shall first recall some notations and collect some basic facts in a preliminaries section, then some key lemmas used to prove our main rigidity theorems are given in section 3. Section 4 is devoted to proving some uniqueness theorems and their corollaries concerning hypersurfaces in semi-Riemannian warped product of constant sectional curvature. Finally, in section 5, we obtain some generalizations of the results proved in section 4 concerning hypersurfaces in semi-Riemannian warped products. The Riemannian version of Theorem 1.1 and 1.2 are given in section 4 and 5 respectively. Some applications of our main results on some physical models are also presented in section 4 and 5 respectively.

\section{Preliminaries}

In this section, following $[5,6]$ we shall recall some basic notations and facts that will appear along this paper. We first introduce some notations on Riemannian immersions in semi-Riemannian manifolds.

Let $\bar{M}^{n+1}$ be a connected semi-Riemannian manifold with metric $\bar{g}=\langle$, for index $\nu \leq 1$ and $\bar{\nabla}$ be the semi-Riemannian connection. In what follows, we consider Riemannian immersion $\psi: \Sigma^{n} \rightarrow \bar{M}^{n+1}$, and we orient $\Sigma^{n}$ by the choice of a unit normal vector field $N$ on it. We denote by $A$ the shape operator of $\psi$. For $0 \leq r \leq n$, let $S_{r}(p)$ be the $r$-th elementary symmetric function of the eigenvalues of $A_{p}$ for $p \in \Sigma^{n}$. Then $S_{r}: \Sigma^{n} \rightarrow \mathbb{R}$ is given as follows

$$
\operatorname{det}(t I-A)=\sum_{k=0}^{n}(-1)^{k} S_{k} t^{n-k}
$$


where $S_{0}=1$ by the definition. If $p \in \Sigma^{n}$ and $\left\{e_{i}\right\}$ is a basis of $T_{p} \Sigma^{n}$ formed by eigenvectors of $A_{p}$ with corresponding eigenvalues $\left\{\lambda_{k}\right\}$, one can immediately get

$$
S_{r}=\sigma_{r}\left(\lambda_{1}, \cdots, \lambda_{n}\right)
$$

where $\sigma_{r} \in \mathbb{R}\left[X_{1}, \cdots, X_{n}\right]$ is the $r$-th elementary symmetric polynomial on the indeterminates $X_{1}, \cdots, X_{n}$. Also, for $0 \leq r \leq n$ we define the $r$-th mean curvature $H_{r}$ of $\psi$ by

$$
C_{n}^{r} H_{r}=\epsilon_{N}^{r} S_{r}=\sigma_{r}\left(\epsilon_{N} \lambda_{1}, \cdots, \epsilon_{N} \lambda_{n}\right) .
$$

It is easy to see that $H_{0}=1$ and $H_{1}$ is the usual mean curvature $H$ of $\Sigma^{n}$. We also notice that the Hilbert-Schmidt norm of the shape operator $A$ of $\Sigma^{n}$ is given by

$$
|A|^{2}=n^{2} H^{2}-n(n-1) H_{2} .
$$

We may define the $r$-th Newton transformation $P_{r}$ on $\Sigma^{n}$ by setting $P_{0}=I$ (the identity operator) for $0 \leq r \leq n$ via the recurrence relation

$$
P_{r}=\epsilon_{N}^{r} S_{r} I-\epsilon_{N} A P_{r-1} .
$$

A trivial induction shows that

$$
P_{r}=\epsilon_{N}^{r}\left(S_{r} I-S_{r-1} A+S_{r-2} A^{2}-\cdots+(-1)^{r} A^{r}\right),
$$

so that the Cayley-Hamilton theorem gives $P_{n}=0$. Moreover, since $P_{r}$ is a polynomial in $A$ for every $r$, it is also self-adjoint and commutes with $A$. Therefore, all basis of $T_{p} \Sigma^{n}$ diagonalizing $A$ at $p \in \Sigma^{n}$ also diagonalize all of the $P_{r}$ at $p$. Let $\left\{e_{i}\right\}$ be such the basis. Denote by $A_{i}$ the restriction of $A$ to $\left\langle e_{i}\right\rangle^{\perp} \subset T_{p} \Sigma^{n}$, it is easy to see that

$$
\operatorname{det}\left(t I-A_{i}\right)=\sum_{k=0}^{n}(-1)^{k} S_{k}\left(A_{i}\right) t^{n-1-k},
$$

where

$$
S_{k}\left(A_{i}\right)=\sum_{\substack{1 \leq j_{1}<\cdots<j_{k} \leq n \\ j_{1} \cdots j_{k} \neq i}} \lambda_{j_{1}} \cdots \lambda_{j_{k}} .
$$

From [8], it is also immediate to check that $P_{r} e_{i}=\epsilon_{N}^{r} S_{r}\left(A_{i}\right) e_{i}$, then an easy computation gives the following result.

Lemma 2.1 (Lemma 2.1 of [8]). With above notations, the following formulas hold:

(a) $S_{r}\left(A_{i}\right)=S_{r}-\lambda_{i} S_{r-1}\left(A_{i}\right)$. 
(b) $\operatorname{tr}\left(P_{r}\right)=\epsilon_{N}^{r} \sum_{i=1}^{n} S_{r}\left(A_{i}\right)=\epsilon_{N}^{r}(n-r) S_{r}=b_{r} H_{r}$

(c) $\operatorname{tr}\left(A P_{r}\right)=\epsilon_{N}^{r} \sum_{i=1}^{n} \lambda_{i} S_{r}\left(A_{i}\right)=\epsilon_{N}^{r}(r+1) S_{r+1}=\epsilon_{N} b_{r} H_{r+1}$.

(d) $\operatorname{tr}\left(A^{2} P_{r}\right)=\epsilon_{N}^{r} \sum_{i=1}^{n} \lambda_{i}^{2} S_{r}\left(A_{i}\right)=\epsilon_{N}^{r}\left(S_{1} S_{r+1}-(r+2) S_{r+2}\right)$,

where $b_{r}=(n-r) C_{n}^{r}$.

Associated to each Newton transformation $P_{r}$ one has the second order linear differential operator $L_{r}: \mathcal{D}\left(\Sigma^{n}\right) \rightarrow \mathcal{D}\left(\Sigma^{n}\right)$ given by

$$
L_{r}(f)=\operatorname{tr}\left(P_{r} \circ \operatorname{Hess} f\right) \text {, }
$$

where $\mathcal{D}\left(\Sigma^{n}\right)$ denotes the set of all smooth functions on $\Sigma^{n}$. In particular, $L_{0}=\Delta$ and from [10] we know that if $\bar{M}$ has constant sectional curvature, then $L_{r}(f)=\operatorname{div}\left(P_{r} \nabla f\right)$, where div denotes the divergence on $\Sigma^{n}$.

For a smooth function $\varphi: \mathbb{R} \rightarrow \mathbb{R}$ and $h \in \mathcal{D}\left(\Sigma^{n}\right)$, it follows from the properties of the Hessian of functions that

$$
L_{r}(\varphi \circ h)=\varphi^{\prime}(h) L_{r}(h)+\varphi^{\prime \prime}(h)\left\langle P_{r} \nabla h, \nabla h\right\rangle .
$$

Now, we give some facts on semi-Riemannian warped products. Let $M^{n}$ be a connected, $n$-dimensional $(n \geq 2)$ oriented Riemannian manifold, $I \subseteq \mathbb{R}$ an open interval and $f: I \rightarrow \mathbb{R}$ a positive smooth function. We consider the product differential manifold $I \times M^{n}$ and denote by $\pi_{I}$ and $\pi_{M}$ the projections onto the base $I$ and the fiber $M^{n}$, respectively. A particular class of semiRiemannian manifolds is the one obtained by furnishing $I \times M^{n}$ with the metric

$$
\langle v, w\rangle_{p}=\epsilon\left\langle\left(\pi_{I}\right)_{*} v,\left(\pi_{I}\right)_{*} w\right\rangle+\left(f \circ \pi_{I}(p)\right)^{2}\left\langle\left(\pi_{M}\right)_{*} v,\left(\pi_{M}\right)_{*} w\right\rangle
$$

for all $p \in \bar{M}^{n+1}$ and all $v, w \in T_{p} \bar{M}^{n+1}$, where $\epsilon=\epsilon_{\partial_{t}}$ and $\partial_{t}$ is the standard unit vector field tangent to $I$ and $f$ is known as the warping function and we denote the space by $\bar{M}^{n+1}=\epsilon I \times{ }_{f} M^{n}$. In particular, $-I \times{ }_{f} M^{n}$ is called a Robertson-Walker spacetime if $M^{n}$ has constant sectional curvature. According to Proposition 42 of [19], we know that a generalized Robertson-Walker spacetime has constant sectional curvature $\bar{k}$ if and only if, the Riemannian fiber $M^{n}$ has constant sectional curvature $k$ and the warping function $f$ satisfies the following differential equation

$$
\frac{f^{\prime \prime}}{f}=\bar{k}=\frac{f^{\prime 2}+k}{f^{2}} .
$$

It follows from [17] that the vector field $\left(f \circ \pi_{I}\right) \partial_{t}$ is conformal and closed (in this sense that its dual 1-form is closed) with conformal factor $\phi=f^{\prime} \circ \pi_{I}$, 
where the prime denotes differentiation with respect to $t \in I$. For $t_{0} \in I$, we orient the slice $\Sigma_{t_{0}}^{n}:=\left\{t_{0}\right\} \times M^{n}$ by using the unit normal vector field $\partial_{t}$, then from $[6,17]$ we know that $\Sigma_{t_{0}}^{n}$ has constant r-th mean curvature $H_{r}=-\epsilon\left(\frac{f^{\prime}\left(t_{0}\right)}{f\left(t_{0}\right)}\right)^{r}$ with respect to $\partial_{t}$.

A smooth immersion $\psi: \Sigma^{n} \rightarrow \epsilon I \times_{f} M^{n}$ of an $n$-dimensional connected manifold $\Sigma^{n}$ is said to be a spacelike hypersurface if the induced metric via $\psi$ is a Riemannian metric on $\Sigma^{n}$. Let $\psi$ be a Riemannian immersion with $\Sigma^{n}$ oriented by the unit vector field $N$, one obviously has $\epsilon=\epsilon \partial_{t}=\epsilon_{N}$. We denote by $h$ the vertical (height) function naturally attached to $\Sigma^{n}$ defined by $h=\left.\left(\pi_{I}\right)\right|_{\Sigma^{n}}$.

We denote by $\bar{\nabla}$ and $\nabla$ the gradient with respect to the metrics of $\epsilon I \times_{f} M^{n}$ and $\Sigma^{n}$ respectively. Then by a simple computation we have the gradient of $\pi_{I}$ on $\epsilon I \times{ }_{f} M^{n}$ which is given by

$$
\bar{\nabla} \pi_{I}=\epsilon\left\langle\bar{\nabla} \pi_{I}, \partial_{t}\right\rangle=\epsilon \partial_{t}
$$

and the gradient of $h$ on $\Sigma^{n}$ is given by

$$
\nabla h=\left(\bar{\nabla} \pi_{I}\right)^{\top}=\epsilon \partial_{t}^{\top}=\epsilon \partial_{t}-\left\langle N, \partial_{t}\right\rangle N .
$$

In particular, we have

$$
|\nabla h|^{2}=\epsilon\left(1-\left\langle N, \partial_{t}\right\rangle^{2}\right)
$$

where $|\cdot|$ denotes the norm of a vector field on $\Sigma^{n}$.

\section{$3 \quad$ Key Lemmas}

We give some important lemmas in order to prove our main theorems. First, following a simple computation we have

$$
\begin{aligned}
L_{r}(f) & =\operatorname{tr}\left(P_{r} \circ \operatorname{Hess} f\right)=\sum_{i=1}^{n}\left\langle P_{r}\left(\nabla_{e_{i}} \nabla f\right), e_{i}\right\rangle \\
& =\sum_{i=1}^{n}\left\langle\nabla_{e_{i}} \nabla f, P_{r}\left(e_{i}\right)\right\rangle=\sum_{i=1}^{n}\left\langle\nabla_{P_{r}\left(e_{i}\right)} \nabla f, e_{i}\right\rangle=\operatorname{tr}\left(\operatorname{Hess} f \circ P_{r}\right),
\end{aligned}
$$

where $\left\{e_{1}, \cdots, e_{n}\right\}$ is a local orthonormal frame on $\Sigma^{n}$. It follows from [10] that

$$
\begin{aligned}
\operatorname{div}_{\Sigma}\left(P_{r}(\nabla f)\right) & =\sum_{i=1}^{n}\left\langle\left(\nabla_{e_{i}} P_{r}\right) \nabla f, e_{i}\right\rangle+\sum_{i=1}^{n}\left\langle P_{r}\left(\nabla_{e_{i}} \nabla f\right), e_{i}\right\rangle \\
& =\left\langle\operatorname{div} P_{r}, \nabla f\right\rangle+L_{r}(f)
\end{aligned}
$$


where the divergence of $P_{r}$ on $\Sigma^{n}$ is given by

$$
\operatorname{div} P_{r}=\operatorname{tr}\left(\nabla P_{r}\right)=\sum_{i=1}^{n}\left(\nabla_{e_{i}} P_{r}\right)\left(e_{i}\right)
$$

From relation (12) we know that the operator $L_{r}$ is elliptic if and only if $P_{r}$ is positive definite. Notice that $L_{0}=\Delta$ is always elliptic. The following two lemmas were proved by L. J. Alías and A. G. Colares [2] in which the authors gave some geometric conditions for guaranteeing the ellipticity of $L_{1}$ and $L_{r}$ for $2 \leq r \leq n$, respectively.

Lemma 3.1 (Lemma 3.2 of [2]). Let $\psi: \Sigma^{n} \rightarrow \bar{M}^{n+1}$ be a Riemannian immersion in a semi-Riemannian manifold $\bar{M}^{n+1}$. If $H_{2}>0$ on $\Sigma^{n}$, then $L_{1}$ is elliptic or, equivalently, $P_{1}$ is positive definite for an appropriate choice of the Gauss map N.

Lemma 3.2 (Lemma 3.3 of [2]). Let $\psi: \Sigma^{n} \rightarrow \bar{M}^{n+1}$ be a Riemannian immersion in a semi-Riemannian manifold $\bar{M}^{n+1}$. If there exists an elliptic point of $\Sigma^{n}$, with respect to an appropriate choice of the Gauss map $N$, and $H_{r+1}>0$ on $\Sigma^{n}$ for $2 \leq r \leq n-1$, then for all $1 \leq k \leq r$ the operator $L_{k}$ is elliptic or, equivalently, $P_{k}$ is positive definite (for an appropriate choice of the Gauss map $N$, if $k$ is odd).

Notice that when we say $p_{0} \in \Sigma^{n}$ being an elliptic point in a semiRiemannian immersion $\psi: \Sigma^{n} \rightarrow \bar{M}^{n+1}$ into a semi-Riemannian manifold $\bar{M}^{n+1}$, we mean that all principal curvatures $\lambda_{i}\left(p_{0}\right)$ of this point have the same sign. Moreover, we also need a sufficient condition to guarantee the existence of an elliptic point in Riemannian immersions. The following result follows from A. G. Colares and H. F. de Lima [13], which is the semi-Riemannian version of Lemma 5.4 of L. J. Alías, A. Brasil Jr and A. G. Colares [1].

Lemma 3.3 (Lemma 5.3 of [13]). Let $\bar{M}^{n+1}=\epsilon I \times{ }_{f} M^{n}$ be a semi-Riemannian warped product manifold and $\psi: \Sigma^{n} \rightarrow \bar{M}^{n+1}$ a Riemannian immersion. If $-\epsilon f(h)$ attains a local minimum at some $p \in \Sigma^{n}$ such that $f^{\prime}(h)(p) \neq 0$, then $p$ is an elliptic point for $\Sigma^{n}$.

Moreover, we also need some properties on operator $L_{r}$. Notice that F. Camargo, A. Camimha and H. F. de Lima [12] proved the following lemma. In the Lorentzian setting, the following result is just a particular case of the one obtained by A. J. Alías and A. G. Colares in Lemma 4.1 of [2]. 
Lemma 3.4 (Lemma 2.2 of [12]). Let $\psi: \Sigma^{n} \rightarrow \epsilon I \times_{f} M^{n}$ be a Riemannian immersion in a semi-Riemannian warped product manifold. If $h=\left.\left(\pi_{I}\right)\right|_{\Sigma^{n}}$ : $\Sigma^{n} \rightarrow I$ is the height function of $\Sigma^{n}$, then

$$
L_{r}(h)=(\ln f)^{\prime}\left(\epsilon \operatorname{tr} P_{r}-\left\langle P_{r} \nabla h, \nabla h\right\rangle\right)+\left\langle N, \partial_{t}\right\rangle \operatorname{tr}\left(A P_{r}\right) .
$$

Using equations (6.2) and (6.16) of [2], H. F. de Lima and H. R. de Lima [14] obtained the following result.

Lemma 3.5 (Lemma 3.4 of [14]). Let $\psi: \Sigma^{n} \rightarrow \epsilon I \times_{f} M^{n}$ be a Riemannian immersion in a semi-Riemannian warped product manifold. If $h=\left.\left(\pi_{I}\right)\right|_{\Sigma^{n}}$ : $\Sigma^{n} \rightarrow I$ is the height function of $\Sigma^{n}$, then

$$
\begin{aligned}
& \left\langle\operatorname{div}_{\Sigma^{n}} P_{1}, \nabla h\right\rangle \\
= & -\epsilon\left(\operatorname{Ric}_{M^{n}}\left(N^{*}, N^{*}\right)+\epsilon(n-1)(\ln f)^{\prime \prime}(h)|\nabla h|^{2}\right)\left\langle N, \partial_{t}\right\rangle,
\end{aligned}
$$

where $\operatorname{Ric}_{M^{n}}$ denotes the Ricci curvature tensor of the fiber $M^{n}$ and $N^{*}=$ $N-\epsilon\left\langle N, \partial_{t}\right\rangle \partial_{t}$ is the projection of the unit normal vector field $N$ of $\Sigma^{n}$ onto $M^{n}$. Moreover, if the fiber $M^{n}$ has constant sectional curvature $k$, then

$$
\left\langle\operatorname{div}_{\Sigma^{n}} P_{r}, \nabla h\right\rangle=-\epsilon(n-r)\left(\frac{k}{f^{2}(h)}+\epsilon(\ln f)^{\prime \prime}(h)\right)\left\langle P_{r-1} \nabla h, \nabla h\right\rangle\left\langle N, \partial_{t}\right\rangle .
$$

In view of Lemma 3.4 and Lemma 3.5, and making use of equation (6), we obtain the following key lemma.

Lemma 3.6. Let $\psi: \Sigma^{n} \rightarrow \epsilon I \times_{f} M^{n}$ be a spacelike hypersurface in a semiRiemannian warped product manifold whose fiber $M^{n}$ has constant sectional curvature $k$. Denoted by $h=\left.\left(\pi_{I}\right)\right|_{\Sigma^{n}}: \Sigma^{n} \rightarrow I$ the height function of $\Sigma^{n}$, if

$$
\sigma(t)=\int_{t_{0}}^{t} f(s) d s
$$

then we obtain

$$
\begin{aligned}
& \operatorname{div}_{\Sigma^{n}}\left(P_{r}(\nabla \sigma(h))\right) \\
= & \epsilon b_{r}\left(f^{\prime}(h) H_{r}+f(h) H_{r+1}\left\langle N, \partial_{t}\right\rangle\right) \\
& -\epsilon(n-r) f(h)\left(\frac{k}{f^{2}(h)}+\epsilon(\ln f)^{\prime \prime}(h)\right)\left\langle P_{r-1} \nabla h, \nabla h\right\rangle\left\langle N, \partial_{t}\right\rangle .
\end{aligned}
$$

Proof. It follows from relation (6) that

$$
L_{r}(\sigma(h))=f(h) L_{r}(h)+f^{\prime}(h)\left\langle P_{r}(\nabla h), \nabla h\right\rangle .
$$


Applying Lemma 2.1 and Lemma 3.4 on relation (19) implies that

$$
\begin{aligned}
L_{r}(\sigma(h))= & f(h)\left((\ln f)^{\prime}\left(\epsilon b_{r} H_{r}-\left\langle P_{r} \nabla h, \nabla h\right\rangle\right)+\epsilon\left\langle N, \partial_{t}\right\rangle b_{r} H_{r+1}\right) \\
& +f^{\prime}(h)\left\langle P_{r}(\nabla h), \nabla h\right\rangle \\
= & \epsilon b_{r}\left(f^{\prime}(h) H_{r}+f(h) H_{r+1}\left\langle N, \partial_{t}\right\rangle\right) .
\end{aligned}
$$

Replacing $f(h)$ by $\sigma(h)$ in $(12)$, we have

$$
\operatorname{div}_{\Sigma}\left(P_{r}(\nabla \sigma(h))\right)=f(h)\left\langle\operatorname{div} P_{r}, \nabla h\right\rangle+L_{r}(\sigma(h)) .
$$

Thus, our proof follows from Lemma 3.5, (20) and (21).

\section{Warped products of constant sectional curvature}

According to $[7,20,21]$, we may say that a spacelike hypersurface $\psi: \Sigma^{n} \rightarrow$ $\epsilon I \times{ }_{f} M^{n}$ is bounded away from the future infinity of $\epsilon I \times{ }_{f} M^{n}$ if there exists $\bar{t} \in I$ such that

$$
\psi\left(\Sigma^{n}\right) \subset\left\{(t, p) \in \epsilon I \times_{f} M^{n}: t \leq \bar{t}\right\} .
$$

Analogously, a spacelike hypersurface $\psi: \Sigma^{n} \rightarrow \epsilon I \times{ }_{f} M^{n}$ is said to be bounded away from the past infinity of $\epsilon I \times{ }_{f} M^{n}$ if there exists $\underline{t} \in I$ such that

$$
\psi\left(\Sigma^{n}\right) \subset\left\{(t, p) \in \epsilon I \times_{f} M^{n}: t \geq \underline{t}\right\} .
$$

Finally, $\Sigma^{n}$ is said to be bounded away from the infinity of $\epsilon I \times_{f} M^{n}$ if it is both bounded away from the past and future infinity of $\epsilon I \times{ }_{f} M^{n}$.

Lemma 4.1 (Corollary 1 of [11]). Let $\bar{M}^{n+1}$ has constant sectional curvature, and $\psi: \Sigma^{n} \rightarrow \bar{M}^{n+1}$ be a complete Riemannian immersion with bounded second fundamental form. Let $g: \Sigma^{n} \rightarrow \mathbb{R}$ be a smooth function such that $|\nabla g| \in \mathcal{L}\left(\Sigma^{n}\right)$. If $L_{r} g$ does not change sign on $\Sigma^{n}$, then $L_{r} g=0$ on $\Sigma^{n}$.

Now, we give our main uniqueness theorems for spacelike hypersurfaces immersed in Lorentzian warped product. Assuming that $N$ is the orientation of the spacelike hypersurface $\psi: \Sigma^{n} \rightarrow-I \times_{f} M^{n}$ and its angle function satisfies $\left\langle N, \partial_{t}\right\rangle<0$, then, by applying the reverse Cauchy-Schwarz inequality, we obtain

$$
\left\langle N, \partial_{t}\right\rangle \leq-1<0
$$

Proof of Theorem 1.1. Letting $\epsilon=-1$, then it follows from (20) that

$$
L_{r}(\sigma(h))=-b_{r}\left(f^{\prime}(h) H_{r}+f(h) H_{r+1}\left\langle N, \partial_{t}\right\rangle\right) .
$$


Noting that both $H_{r}$ and $H_{r+1}$ are positive, then, making use of the assumption (1) and (22) in (23) we obtain

$$
\begin{aligned}
L_{r}(\sigma(h)) & =-b_{r} H_{r} f(h)\left(\frac{f^{\prime}}{f}(h)+\frac{H_{r+1}}{H_{r}}\left\langle N, \partial_{t}\right\rangle\right) \\
& \geq-b_{r} H_{r} f(h)\left(\frac{f^{\prime}}{f}(h)-\frac{H_{r+1}}{H_{r}}\right) \geq 0 .
\end{aligned}
$$

On the other hand, since the spacelike hypersurface $\Sigma^{n}$ is bounded away from the infinity of $-I \times{ }_{f} M^{n}$, then the height function $h$ is also bounded on $\Sigma^{n}$. Also, we have

$$
|\nabla \sigma(h)|=f(h)|\nabla h|
$$

this means that $|\nabla \sigma(h)|$ is integrable since that $|\nabla h|$ is integrable on $\Sigma^{n}$. The above arguments guarantees that Lemma 4.1 is applicable, then applying Lemma 4.1 on the smooth function $\sigma(h)$ on $\Sigma^{n}$ we have

$$
L_{r}(\sigma(h))=0
$$

Putting the above equation into (32) and noting that $b_{r}$ is positive, then we obtain $f^{\prime}(h) H_{r}+f(h) H_{r+1}\left\langle N, \partial_{t}\right\rangle=0$, thus, making use of inequality (1) in this equation gives that

$$
\frac{f^{\prime}}{f}=-\left\langle N, \partial_{t}\right\rangle \frac{H_{r+1}}{H_{r}} \geq-\left\langle N, \partial_{t}\right\rangle \frac{f^{\prime}}{f}>0 .
$$

Notice that hypothesis (1) guarantees that $\frac{f^{\prime}}{f}>0$ on $I$, then it follows from the above inequality that $-\left\langle N, \partial_{t}\right\rangle \leq 1$, comparing this inequality with inequality (22) we obtain equation $\left\langle N, \partial_{t}\right\rangle=-1$. Finally, using $\epsilon=-1$ and $\left\langle N, \partial_{t}\right\rangle=-1$ in relation (10) gives that

$$
|\nabla h|^{2}=-\left(1-\left\langle N, \partial_{t}\right\rangle^{2}\right) \equiv 0
$$

Thus, we prove that $\Sigma^{n}$ is a slice of $\bar{M}^{n+1}$.

Remark 4.1. Theorem 5.4 of [13] attains the same conclusion as our Theorem 1.1, however, in our hypotheses we do not need the condition that the warping function $f$ has convex logarithm.

Next we consider the steady state-type spacetime, i.e., the Lorentzian warped product $-\mathbb{R} \times_{e^{t}} M^{n}$, where the fiber $M^{n}$ is an $n$-dimensional complete and connected Riemannian manifold. The importance of considering $\mathcal{H}^{n+1}=-\mathbb{R} \times e_{e^{t}} \mathbb{R}^{n}$ comes from the fact that, in cosmology, $\mathcal{H}^{4}$ is the steady model of the universe proposed by H. Bondi and T. Gold [9], and F. Holy [15]. 
Moreover, following [12] we see that in physical context the steady state space appears naturally as an exact solution for the Einstein equations, being a cosmological model where matter is supposed to travel along geodesic normal to horizontal hyperplanes. We refer the reader to [?] for an alternative description of the steady state space $\mathcal{H}^{n+1}$. Consider a steady state-type spacetime $-\mathbb{R} \times{ }_{e^{t}} M^{n}$ of constant sectional curvature, then from (7) we see that the fiber $M^{n}$ of $-\mathbb{R} \times e^{t} M^{n}$ is of constant sectional zero. Thus, the following result is true.

Corollary 4.1 (Theorem 3.6 of [12]). Let $\bar{M}^{n+1}=-\mathbb{R} \times{ }_{e^{t}} M^{n}$ be a steady state-type spacetime whose fiber is of constant sectional curvature zero. Let $\psi$ : $\Sigma^{n} \rightarrow \bar{M}^{n+1}$ be a complete and connected spacelike hypersurface with bounded second fundamental form and bounded away from the infinity of $\bar{M}^{n+1}$. Suppose that the $r$-th mean curvatures satisfy $0<H_{r} \leq H_{r+1}$ for some $1 \leq r \leq$ $n-1$. If $|\nabla h| \in \mathcal{L}\left(\Sigma^{n}\right)$ on $\Sigma^{n}$, then $\Sigma^{n}$ is a slice of $\bar{M}^{n+1}$.

Now, we give the uniqueness theorems (which is Riemannian version of Theorem 1.1) for spacelike hypersurfaces immersed in Riemannian warped product. Assuming that $N$ is the orientation of the spacelike hypersurface $\psi: \Sigma^{n} \rightarrow I \times_{f} M^{n}$ and its angle function satisfies $\left\langle N, \partial_{t}\right\rangle<0$, then, by applying the Cauchy-Schwarz inequality, we obtain

$$
-1 \leq\left\langle N, \partial_{t}\right\rangle<0
$$

Theorem 4.1. Let $\bar{M}^{n+1}=I \times_{f} M^{n}$ be a Riemannian warped product of constant sectional curvature. Let $\psi: \Sigma^{n} \rightarrow \bar{M}^{n+1}$ be a complete and connected spacelike hypersurface with bounded second fundamental form and bounded away from the infinity of $\bar{M}^{n+1}$. Suppose that $H_{r}$ and $H_{r+1}$ are positive for some $1 \leq r \leq n-1$ such that

$$
\frac{H_{r+1}}{H_{r}} \leq \frac{f^{\prime}}{f}(h)
$$

If h has local maximum on $\Sigma^{n}$ and $|\nabla h| \in \mathcal{L}\left(\Sigma^{n}\right)$, then $\Sigma^{n}$ is a slice of $\bar{M}^{n+1}$.

Proof. Letting $\epsilon=1$, then it follows from (20) that

$$
L_{r}(\sigma(h))=b_{r}\left(f^{\prime}(h) H_{r}+f(h) H_{r+1}\left\langle N, \partial_{t}\right\rangle\right) .
$$

Noting that both $H_{r}$ and $H_{r+1}$ are positive, then, making use of assumption 
(29) and (30) in (31) we obtain

$$
\begin{aligned}
L_{r}(\sigma(h)) & =b_{r} H_{r} f(h)\left(\frac{f^{\prime}}{f}(h)+\frac{H_{r+1}}{H_{r}}\left\langle N, \partial_{t}\right\rangle\right) \\
& \geq b_{r} H_{r} f(h)\left(\frac{f^{\prime}}{f}(h)-\frac{H_{r+1}}{H_{r}}\right) \\
& \geq 0 .
\end{aligned}
$$

On the other hand, since the spacelike hypersurface $\Sigma^{n}$ is bounded away from the infinity of $I \times{ }_{f} M^{n}$, then the height function $h$ is also bounded on $\Sigma^{n}$. Also, we have $|\nabla \sigma(h)|=f(h)|\nabla h|$, this means that $|\nabla \sigma(h)|$ is integrable since that $|\nabla h|$ is integrable on $\Sigma^{n}$. The above arguments implies that Lemma 4.1 is applicable, then applying Lemma 4.1 on the smooth function $\sigma(h)$ on $\Sigma^{n}$ we have

$$
L_{r}(\sigma(h))=0 \text {. }
$$

Putting the above equation into (32) and noting that $b_{r}$ is positive, then we obtain $f^{\prime}(h) H_{r}+f(h) H_{r+1}\left\langle N, \partial_{t}\right\rangle=0$, thus, making use of inequality (30) in this equation gives that

$$
0<\frac{f^{\prime}}{f}=-\left\langle N, \partial_{t}\right\rangle \frac{H_{r+1}}{H_{r}} \leq-\left\langle N, \partial_{t}\right\rangle \frac{f^{\prime}}{f} .
$$

Notice that hypothesis (30) guarantees that $\frac{f^{\prime}}{f}>0$ on $I$, then it follows from the above inequality that $-\left\langle N, \partial_{t}\right\rangle \geq 1$, comparing this inequality with inequality (29) we obtain equation $\left\langle N, \partial_{t}\right\rangle=-1$. Finally, making use of $\epsilon=1$ and $\left\langle N, \partial_{t}\right\rangle=-1$ in (10) gives that

$$
|\nabla h|^{2}=1-\left\langle N, \partial_{t}\right\rangle^{2} \equiv 0 .
$$

Thus, we prove that $\Sigma^{n}$ is a slice of $\bar{M}^{n+1}$.

The hyperbolic-type space is defined by $\mathbb{R} \times_{e^{t}} M^{n}$, where $M^{n}$ is a complete connected Riemannian manifold. The motivation for investigating the hyperbolic-type space $\mathbb{R} \times_{e^{t}} M^{n}$ comes from the fact that, the $(n+1)$-dimensional hyperbolic space $\mathbb{H}^{n+1}$ is isometric to $\mathbb{R} \times e^{t} \mathbb{R}^{n}$. Noting that an explicit isometry between the half-space model and this hyperbolictype model has been pointed out by L. J. Alías and M. Dajczer in [3].

Now letting the warping function be $f=e^{t}$ for $t \in \mathbb{R}$, then the following result follows from Theorem 4.1 and Lemma 5.2.

Corollary 4.2 (Theorem 3.7 of [12]). Let $\bar{M}^{n+1}=I \times{ }_{e^{t}} M^{n}$ be a hyperbolictype space whose fiber is of constant sectional curvature zero. Let $\psi: \Sigma^{n} \rightarrow$ 
$\bar{M}^{n+1}$ be a complete and connected spacelike hypersurface with bounded second fundamental form and bounded away from the infinity of $\bar{M}^{n+1}$. Suppose that the $r$-th mean curvatures satisfy $0<H_{r+1} \leq H_{r}$ for some $1 \leq r \leq n-1$. If $|\nabla h| \in \mathcal{L}\left(\Sigma^{n}\right)$ on $\Sigma^{n}$, then $\Sigma^{n}$ is a slice of $\bar{M}^{n+1}$.

Remark 4.2. We refer the reader to S. Montiel [18] and B. O'Neill [19] for some examples of semi-Riemannian warped products whose warping functions are not necessarily to have convex logarithm. Without requiring the condition that the warping function $f$ has convex logarithm, Theorem 4.1 attains the same conclusion as Theorem 5.8 of [13]. That is, our Theorem 4.1 is an extension of Theorem 5.8 of [13].

\section{Semi-Riemannian warped products}

In this section, in order to prove our main theorems, we shall make use of the following lemma obtained by A. Caminha [10]. Notice that the following lemma extends a result of S. T. Yau [22] on a version of Stokes theorem for an $n$-dimensional complete and noncompact Riemannian manifold.

Lemma 5.1 (Proposition 2.1 of [10]). Let $X$ be a smooth vector field on the $n$ dimensional complete, noncompact, oriented Riemannian manifold $M^{n}$, such that $\operatorname{div}_{M^{n}} X$ does not change sign on $M^{n}$. If $|X| \in \mathcal{L}\left(M^{n}\right)$, then $\operatorname{div}_{\mathrm{M}^{\mathrm{n}}}=0$.

By using Proposition 42 of [19] proved by B. O'Neill, we obtained the following result. Here we omit the proof of Lemma 5.2 since that it is similar to that of Corollary 2.4 of [16] proved by T. H. Kang.

Lemma 5.2. Let $\bar{M}^{n+1}=\epsilon I \times_{f} M^{n}$ be a semi-Riemannian warped product whose fiber is of constant sectional curvature $k$. Then, $\bar{M}^{n+1}$ is of constant sectional curvature if and only if the warping function satisfies

$$
\epsilon f f^{\prime \prime}-\epsilon f^{\prime 2}+k=0 \text {. }
$$

Proof of Theorem 1.2. We assume that $N$ is the orientation of the spacelike hypersurface $\psi: \Sigma^{n} \rightarrow-I \times{ }_{f} M^{n}$ and its angle function satisfies $\left\langle N, \partial_{t}\right\rangle<0$, then inequality (22) folds. Letting $\epsilon=-1$, then it follows from relation (18) that

$$
\begin{aligned}
& \operatorname{div}_{\Sigma^{n}}\left(P_{r}(\nabla \sigma(h))\right) \\
= & -b_{r} f(h) H_{r}\left(\frac{f^{\prime}(h)}{f(h)}+\frac{H_{r+1}}{H_{r}}\left\langle N, \partial_{t}\right\rangle\right) \\
& +(n-r) f(h) \frac{k-\left(f f^{\prime \prime}(h)-f^{\prime 2}(h)\right)}{f^{2}(h)}\left\langle P_{r-1} \nabla h, \nabla h\right\rangle\left\langle N, \partial_{t}\right\rangle .
\end{aligned}
$$


Next we claim that under the assumption of Theorem 1.2, the Newton transformation $P_{r}$ is positive definite for some $1 \leq r \leq n-1$. In fact, first, if $H_{2}>0$ then by applying Lemma 3.1 we know $P_{1}$ is positive. Otherwise, noticing the assumption (1) that $f^{\prime}(h)$ does not vanish on $\Sigma^{n}$, then applying Lemma 3.3 we see that there exists an elliptic point $p_{0} \in \Sigma^{n}$. Since both $H_{r}$ and $H_{r+1}$ are positive, applying Lemma 3.2 we know that $L_{r}$ is elliptic or, equivalently, $P_{r}$ is positive definite.

From (1) and (2) we see that $\frac{H_{r+1}}{H_{r}}\left\langle N, \partial_{t}\right\rangle \leq-\frac{f^{\prime}(h)}{f(h)}$, this means that the first term of the right hand side of (36) is nonnegative. Together with the assumption (1), (2), the fact that $f$ is always positive on $\Sigma^{n}$ and $P_{r}$ is positive definite on $\Sigma^{n}$, thus, it follows from (36) that

$$
\operatorname{div}_{\Sigma^{n}}\left(P_{r}(\nabla \sigma(h))\right) \geq 0
$$

On the other hand, since $\Sigma^{n}$ is bounded away from the infinity of $M^{n+1}$ and the eigenvalues are continuous functions on $\Sigma^{n}$, and noting that the shape operator $A$ is bounded on $\Sigma^{n}$, then it follows from (4) that $\left|P_{r}\right|$ is bounded on $\Sigma^{n}$. That is, there exists a positive constant $C>0$ such that $\left|P_{r}\right| \leq C$ on $\Sigma^{n}$, which means that $\left|P_{r}(\nabla h)\right| \leq\left|P_{r}\right||\nabla h| \leq C|\nabla h|$. As $|\nabla h| \in \mathcal{L}\left(\Sigma^{n}\right)$ then we obtain

$$
\left|P_{r}(\nabla h)\right| \in \mathcal{L}\left(\Sigma^{n}\right) .
$$

Furthermore, taking into account (37) and (38) and applying Lemma 5.1 to vector field $X=P_{r}(\nabla(\sigma(h)))$ we get that $\operatorname{div}_{\Sigma^{n}}\left(P_{r}(\nabla \sigma(h))\right)=0$. Noticing that both the two terms on the right hand side of (36) are nonnegative in this case, then both the two terms are zero. Consequently, in view of (22) and (1), it follows from $(36)$ that $\left\langle P_{r-1} \nabla h, \nabla h\right\rangle=0$. In fact, if there exists $p \in \Sigma^{n}$ such that $|\nabla h|(p)>0$, by using $\operatorname{div}_{\Sigma^{n}}\left(P_{r}(\nabla \sigma(h))\right)=0$ and the second term of the right hand side of (36) is nonnegative, it follows from the above arguments that $k-\left(f f^{\prime \prime}(h)-f^{\prime 2}(h)\right)=0$. Applying Lemma 5.2 we know that in this case $\bar{M}^{n+1}$ is of constant sectional curvature, then the proof follows from Theorem 1.1. If not, since that $P_{r-1}$ is positive definite on $\Sigma^{n}$ for some $2 \leq r \leq n$, then the above analyses imply that

$$
\nabla h \equiv 0,
$$

i.e., $h$ is a constant on $\Sigma^{n}$. Thus, we prove that $\Sigma^{n}$ is a slice of $-I \times{ }_{f} M^{n}$.

Before giving the Riemannian version of above theorem concerning spacelike hypersurface in Riemannian warped product manifold, we present the following remark.

Remark 5.1. Noticing that $\left\langle N, \partial_{t}\right\rangle \leq-1$ and $P_{r-1}$ is positive definite, then the assumption (1) of [14] does not assures that equation (23) in [14] is non- 
negative. Only if we correct the equation (1) in [14] as follows:

$$
k \leq \inf _{I}\left(f f^{\prime \prime}-f^{\prime 2}\right)
$$

the proof of Theorem 1.1 of [14] can continue and in this case the conclusion of this theorem still holds. However, the proof need the assumption that $\ln f$ is a convex function, i.e., $(\ln f)^{\prime \prime} \geq 0$ on $\Sigma^{n}$.

Theorem 5.1. Let $\bar{M}^{n+1}=I \times_{f} M^{n}$ be a Riemannian warped product whose Riemannian fiber $M^{n}$ has constant sectional curvature $k$ satisfying

$$
k \geq \sup _{I}\left(f^{\prime 2}-f f^{\prime \prime}\right) .
$$

Let $\psi: \Sigma^{n} \rightarrow \bar{M}^{n+1}$ be a complete noncompact and connected spacelike hypersurface with bounded second fundamental form and bounded away from the infinity of $\bar{M}^{n+1}$. Suppose that $H_{r}$ and $H_{r+1}$ are positive for some $1 \leq r \leq n-1$ and satisfy (30). If $h$ has local maximum on $\Sigma^{n}$ and $|\nabla h| \in \mathcal{L}\left(\Sigma^{n}\right)$, then $\Sigma^{n}$ is a slice.

Proof. Assuming that $N$ is the orientation of the spacelike hypersurface $\psi$ : $\Sigma^{n} \rightarrow I \times_{f} M^{n}$ and its angle function satisfies $\left\langle N, \partial_{t}\right\rangle<0$, then (29) holds. In Riemannian case letting $\epsilon=1$, then it follows from (18) that

$$
\begin{aligned}
& \operatorname{div}_{\Sigma^{n}}\left(P_{r}(\nabla \sigma(h))\right) \\
= & b_{r} f(h) H_{r}\left(\frac{f^{\prime}(h)}{f(h)}+\frac{H_{r+1}}{H_{r}}\left\langle N, \partial_{t}\right\rangle\right) \\
& -(n-r) f(h) \frac{k-\left(f^{\prime 2}(h)-f f^{\prime \prime}(h)\right)}{f^{2}(h)}\left\langle P_{r-1} \nabla h, \nabla h\right\rangle\left\langle N, \partial_{t}\right\rangle .
\end{aligned}
$$

Since both $H_{r-1}$ and $H_{r}$ are positive, then (29) implies that $f^{\prime}(h)$ is always positive on $\Sigma^{n}$. As discussed in proof of Theorem 1.2, applying Lemma 3.1, 3.2 and 3.3 we see that the Newton transformation $P_{r}$ is positive definite under the assumptions of Theorem 5.1. Moreover, from (29) and (30) it is easy to see $\frac{H_{r+1}}{H_{r}}\left\langle N, \partial_{t}\right\rangle \geq-\frac{f^{\prime}(h)}{f(h)}$, this means that the first term of the right hand side of (41) is nonnegative. Together with the assumption (29) and (30), and the fact that $f$ is always positive on $\Sigma^{n}$ and $P_{r}$ is positive definite on $\Sigma^{n}$, then we see from (41) that (37) still holds.

On the other hand, notice that the shape operator $A$ is bounded on $\Sigma^{n}$, then it follows from (4) that $\left|P_{r}\right|$ is bounded on $\Sigma^{n}$, i.e., there exists a positive constant $C>0$ such that $\left|P_{r}\right| \leq C$ on $\Sigma^{n}$, which means that $\left|P_{r}(\nabla h)\right| \leq$ $\left|P_{r}\right||\nabla h| \leq C|\nabla h| \in \mathcal{L}\left(\Sigma^{n}\right)$. 
Moreover, taking into account inequality (37) and the fact that $\left|P_{r}(\nabla h)\right| \in$ $\mathcal{L}\left(\Sigma^{n}\right)$, applying Lemma 5.1 to vector field $X=P_{r}(\nabla(\sigma(h)))$ we conclude that $\operatorname{div}_{\Sigma^{n}}\left(P_{r}(\nabla \sigma(h))\right)=0$. Notice that both two terms on the right hand side of (41) are nonnegative, then both the two terms are zero. Consequently, it follows from (29) and (30) that $\left\langle P_{r-1} \nabla h, \nabla h\right\rangle=0$. Since that $P_{r-1}$ is positive definite on $\Sigma^{n}$ for some $2 \leq r \leq n$, then from the above arguments and the proof of Theorem 1.2 we have that

$$
\nabla h \equiv 0,
$$

i.e., $h$ is a constant. This means that $\Sigma^{n}$ is a slice of $I \times{ }_{f} M^{n}$.

As discussed in Remark 4.2, the condition ln $f$ being convex plays an important role in proof of Theorem 1.2 of [14]. Our Theorem 5.1 extends the conclusion of Theorem 1.2 of [14] without requiring this condition. Also, we refer the reader to [5] for an another use of this condition.

Lemma 5.3. Let $\psi: \Sigma^{n} \rightarrow \epsilon I \times{ }_{f} M^{n}$ be a spacelike hypersurface in a semiRiemannian warped product manifold. Denoted by $h=\left.\left(\pi_{I}\right)\right|_{\Sigma^{n}}: \Sigma^{n} \rightarrow I$ the height function of $\Sigma^{n}$, if $\sigma(t)=\int_{t_{0}}^{t} f(s) d s$, then

$$
\begin{aligned}
& \operatorname{div}_{\Sigma^{n}}\left(P_{r}(\nabla \sigma(h))\right) \\
= & -\epsilon f(h)\left(\operatorname{Ric}_{M^{n}}\left(N^{*}, N^{*}\right)+\epsilon(n-1)\left(f f^{\prime \prime}-f^{\prime 2}\right)\left\langle N^{*}, N^{*}\right\rangle_{M^{n}}\right)\left\langle N, \partial_{t}\right\rangle \\
& +\epsilon b_{r}\left(f^{\prime}(h) H_{r}+f(h) H_{r+1}\left\langle N, \partial_{t}\right\rangle\right) .
\end{aligned}
$$

Proof. Noticing that $N^{*}=N-\epsilon\left\langle N, \partial_{t}\right\rangle \partial_{t}$, then it follows from (9) that

$$
\left\langle N^{*}, N^{*}\right\rangle_{M^{n}}=\frac{1}{f^{2}(h)}|\nabla h|^{2} .
$$

Thus, putting (15), (20) and (43) into relation (21) proves (42).

Theorem 5.2. Let $\bar{M}^{n+1}=-I \times_{f} M^{n}$ be a Lorentzian warped product whose Riemannian fiber $M^{n}$ has Ricci curvature Ric satisfying

$$
\operatorname{Ric}_{M^{n}} \geq(n-1) \sup _{I}\left(f f^{\prime \prime}-f^{\prime 2}\right)\langle,\rangle_{M^{n}}
$$

Let $\psi: \Sigma^{n} \rightarrow \bar{M}^{n+1}$ be a complete noncompact and connected spacelike hypersurface with bounded second fundamental form and bounded away from the infinity of $\bar{M}^{n+1}$. Suppose that $H_{r}$ and $H_{r+1}$ are positive for some $1 \leq r \leq n-1$ and satisfy inequality (1). If $h$ has local minimum on $\Sigma^{n}$ and $|\nabla h| \in \mathcal{L}\left(\Sigma^{n}\right)$, then $\Sigma^{n}$ is a slice. 
Proof. In this context, letting $\epsilon=-1$ and hence inequality (44) assures that the first term of the right hand side of (42) is nonnegative. Moreover, inequality (1) assures that the second term of the right hand side of (42) is nonnegative. Thus, the proof follows from Theorem 1.1.

Theorem 5.3. Let $\bar{M}^{n+1}=I \times_{f} M^{n}$ be a Riemannian warped product whose Riemannian fiber $M^{n}$ has Ricci curvature Ric satisfying

$$
\operatorname{Ric}_{M^{n}} \geq(n-1) \sup _{I}\left(f^{\prime 2}-f f^{\prime \prime}\right)\langle,\rangle_{M^{n}}
$$

Let $\psi: \Sigma^{n} \rightarrow \bar{M}^{n+1}$ be a complete noncompact and connected spacelike hypersurface with bounded second fundamental form and bounded away from the infinity of $\bar{M}^{n+1}$. Suppose that $H_{r}$ and $H_{r+1}$ are positive for some $1 \leq r \leq n-1$ and satisfy inequality (30). If h has local maximum on $\Sigma^{n}$ and $|\nabla h| \in \mathcal{L}\left(\Sigma^{n}\right)$, then $\Sigma^{n}$ is a slice of $\bar{M}^{n+1}$.

Proof. In this case, letting $\epsilon=1$ and hence inequality (45) assures that the first term of the right hand side of (42) is nonnegative. Thus, the proof follows from Theorem 5.1.

Acknowledgements: This project was supported by the National Natural Science Foundation of China (No. 11371076 and 11431009).

\section{References}

[1] A. L. Alías, A. Brasil Jr, A. G. Colares, Intergral formulae for spacelike hypersurfaces in conformally stationary spacetimes and applications, Proc. Edinb. Math. Soc., 46 (2003), 465-488.

[2] L. J. Alías, A. G. Colares Uniqueness of spacelike hypersurfaces with constant higher order mean curvature in generalized Robertson-Walker spacetimes, Math. Proc. Camb. Phil. Soc., 143 (2007), 703-729.

[3] L. J. Alías, M. Dajczer, Uniqueness of constant mean curvature surfaces properly immersed in a slab, Comment. Math. Helv., 81 (2006), 653-663.

[4] L. J. Alías, D. Impera, M. Rigoli, Hypersurfaces of constant higher order mean curvature in warped profuct spaces, Trans. Amer. Math. Soc., 365 (2013), 591-621. 
[5] L. J. Alías, D. Impera, M. Rigoli, Spacelike hypersurfaces of constant higher order mean curvature in generalized Robertson-Walker spacetimes, Math. Proc. Camb. Phil. Soc., 152 (2012), 365-383.

[6] L. J. Alías, A. Romero, M. Sánchez, Uniqueness of complete spacelike hypersurfaces with constant mean curvature in generalized RobertsonWalker spacetimes, Gen. Relat. Grav., 27 (1995), 71-84.

[7] C. P. Aquino, H. F. de Lima, Uniqueness of complete hypersurfaces with bounded higher order mean curvatures in semi-Riemannian warped products, Glasgow Math. J., 54 (2012), 201-212.

[8] J. L. M. Barbosa, A. G. Colares, Stability of hypersurfaces with constant r-th mean curvature, Ann. Global Anal. Geom., 15 (1997), 277-297.

[9] H. Bondi, T. Gold, On the generation of magnetism by fluid motion, Monthly Not. Roy. Astr. Soc., 108 (1948), 252-270.

[10] A. Caminha, The geometry of calosed conformal vector fields on Riemannian spaces, Bull. Braz. Math Soc., 42 (2011), 277-300.

[11] A. Caminha, P. Sousa, F. Camargo, Complete foliations of space forms by hypersurfaces, Bull. Braz. Math. Soc., 41 (2010), 339-353.

[12] F. Camargo, A. Caminha, H. F. de Lima, Bernstein-type theorems in semi-Riemannian warped products, Proc. Amer. Math. Soc., 139 (2011), $1841-1850$.

[13] A. G. Colares, H. F. de Lima, Some rigidity theorems in semi-Riemannian warped products, Kodai Math. J., 35 (2012), 268-282.

[14] H. F. de Lima, J. R. de Lima, Complete hypersurfaces immersed in a semi-Riemannian warped product, Differ. Geom. Appl., 30 (2012), 136143.

[15] F. Hoyle, A new model for the expanding universe, Monthly Not. Roy. Astr. Soc., 108 (1948), 372-382.

[16] T. H. Kang, On Lightlike hypersurfaces of a GRW Space-time, Bull. Korean Math. Soc., 49 (2012), 863-874.

[17] S. Montiel, Unicity of constant mean curvature hypersurfaces in some Riemannian manifolds, Indiana Univ. Math. J., 48 (1999), 711-748.

[18] S. Montiel, Complete non-compact spacelike hypersurfaces of constant mean curvature in de Sitter space, J. Math. Soc. Japan, 55 (2003), 915938. 
[19] B. O'Neill, Semi-Riemannian Geometry with Application to Relativity, Academic Press, New York, 1983.

[20] Y. Wang, X. Liu, On complete spacelike hypersurfaces in a semiRiemannian warped product, J. Appl. Math., Volume 2013, Article ID 757041, 8 pages

[21] W. Wang, X. Liu, On Bernstein-type theorems in semi-Riemannian warped products, Adv. Math. Phys., Volume 2013, Article ID 959143, 5 pages

[22] S. T. Yau, Some function-theoretic properties of complete Riemannian manifolds and their applications to geometry, Indiana Univ. Math. J., 25 (1976), 659-670.

Yaning Wang

College of Mathematics and Information Science

Henan Normal University

Xinxiang 453007, Henan, P. R. China

E-mail: wyn051@163.com

Ximin Liu

School of Mathematical Sciences

Dalian University of Technology

Dalian 116024, Liaoning, P. R. China

E-mail: ximinliu@dlut.edu.cn 